\title{
Eye fixation and spatial organization in imagery*
}

\author{
DOUGLAS C. HALL \\ American Institutes for Research, P.O. Box 1113, Palo Alto, California 94302
}

\begin{abstract}
The relationship between eye fixation and spatial organization in imagery was investigated. A spatially organized stimulus consisting of 12 geometric shapes was presented. During recall, eye fixations were allowed to be appropriate to an image of the stimulus, or, alternatively, Ss had to fixate one spatial position. The number of stimulus elements recalled and the latency of the recall were measured. Results indicated that fixating one spatial position tended to disrupt the process of imaging spatial organization, compared to allowing appropriate scanning during recall.
\end{abstract}

There is much debate about the possible role of eye fixations and scanning movements in the retrieval of items from memory. For instance, Hebb (1968) proposes that eye movement has an organizing function in imagery, and Haslerud (1972) suggests that there is a great similarity between the exploration of the external visual field and the internal scanning of memory images when the eyes are closed (p. 95). This suggests that there might be some conflict if internal scanning patterns required for memory retrieval were not compatible with some required simultaneous visual activity in the external world.

This view has recently been supported in a parallel study by the present author, ${ }^{1}$ which showed that recall of tachistoscopic material was accompanied by eye fixations appropriate to the previously presented spatial positions of the items being recalled.

On the other hand, several recent studies have examined eye fixation and visual imagery and have failed to find a significant relationship between them. Bower (1972) found no difference in recall performance when Ss were instructed to generate images linking paired associates under conditions which either permitted eye movement or not. In a similar experiment, Hale and Simpson (1970) found no differences in subjective ratings of the time to construct an image and the vividness of an image. A study comparing eye fixation rates during a variety of visual imagery tasks found that ocular activity did not exceed baseline levels for any of the tasks (Marks, 1972).

These studies have not examined all visual dimensions in imagery that might logically be associated with eye fixation. Some of the studies have examined, but failed to find an association between, eye fixation and the visual dimension of vividness. Another visual dimension, spatial organization, defined as the relative location of elements in two-dimensional space, may be a visual dimension in imagery that is closely associated with eye fixation.

There is evidence that when spatial organization is a salient visual dimension in an imagery task, appropriate eye fixations are elicited. Kahneman (1973) found that

\footnotetext{
*This paper is sponsored by Norman Mackworth, who takes
} full editorial responsibility for its contents.
Ss recalling visual information from a spatial array tended to fixate areas of a blank screen where the information had appeared. Marks $(1972,1974)$ found that Ss tended to fixate appropriate areas of a blank screen when directional cues in the questions prompting recall of visual scenes made spatial organization a salient dimension.

The present experiment examined the relationship between eye fixation and imagery in a task in which spatial organization was a salient visual dimension. Eye fixations were manipulated to produce external patterns appropriate or inappropriate to an internal scanning pattern involved in the spatially organized recall of a visual stimulus.

\section{METHOD}

\section{Subjects}

There were 10 paid undergraduate Ss $(7$ males). They were recruited by notices posted on dormitory bulletin boards. All were naive regarding the purpose of the experiment.

\section{Presentation}

The stimuli were 12 white geometric shapes: triangle, cone, pentagon, square, diamond, oval, cylinder, parallelogram, circle, trapezoid, rectangle, and arc. The shapes were back projected one at a time, each at one of 12 different locations on the screen. The locations where shapes were presented were spaced equally around the screen in a square pattern which subtended 32-46 deg of visual angle. The location at which each shape was presented was varied from trial to trial, as was the presentation sequence. $S$ learned the names of the shapes and identified them before the first trial. No restriction was placed on eye fixation during presentation.

\section{Rehearsal}

After each 3-sec presentation of a shape, a 10-sec rehearsal period was allowed. During this rehearsal period, a display of 13 points of light was presented. Twelve of these lights were spaced equally around the screen in a square pattern that marked the locations at which shapes were presented. One light marked the center of the display area. There were two conditions of rehearsal: (A) Fixation-S had to fixate the central light while rehearsing the locations where shapes had appeared up to that point in the presentation; (B) Scanning-S could look around at the square pattern of 12 lights as he wished. The sequence in which these conditions were imposed was systematically varied between Ss.

\section{Recall}

After presentation of all 12 geometric shapes, the lights 
remained on the display. $S$ was asked to recall the shapes by name, according to which had appeared at each spatially consecutive location, either clockwise or counterclockwise as directed. The starting location was indicated by causing one of the 12 points of light to flash briefly. There were two conditions of recall which corresponded to the conditions imposed during rehearsal: (A) Fixation-If S had fixated the central light during rehearsal, he had to fixate the central light during recall. (B) Scanning-If S had looked around the square pattern of 12 lights during rehearsal, he could look around the lights during recall. S's responses were recorded by the E. A Hunter timer was started when the point of light was flashed, and was stopped when the $S$ arrived back at the starting location after having attempted to recall the shapes in the order of their locations around the screen.

\section{Apparatus}

$\mathrm{S}$ viewed the screen through the wide-angle reflection eye camera (Mackworth, 1968). With this apparatus an image of the display is reflected from S's pupil into the camera. The center of the pupil indicates which part of the display is being looked at by $\mathrm{S}$ at any particular moment. Ss were told that their eye fixations were recorded, and would be checked to see if they were complying with instructions. In fact, however, no film was present in the camera. As a check on compliance the fixations of four Ss were observed by an assistant.

\section{RESULTS}

\section{Main Finding}

When $\mathrm{S}$ was allowed to scan 12 points of light around the periphery of the display area, recall of the shapes in the correct spatial order was significantly better than when $\mathrm{S}$ had to fixate a central point of light.

\section{Eye Fixation and Spatially Organized Recall}

The data were analyzed by means of analyses of variance for single factor experiments having repeated measures (Winer, 1967) and by product-moment correlation. Since performance was measured by number of items recalled and response latency, two analyses of variance were computed, one for each measure. The degree of relationship between measures was analyzed by means of product-moment correlation.

Both analyses of variance revealed significant effects for the recall of geometric shapes. There was a significant effect of conditions (fixation vs scanning) on the number of items recalled $(F=7.33, \mathrm{df}=1 / 9$, $\mathrm{p}<.05)$ and on the latency of the recall $(\mathrm{F}=13.14, \mathrm{df}=$ $1 / 9, p<.01)$. The product-moment correlation between number of items recalled and recall latency was negative $(r=-.65)$ (i.e., recall latency was greater when fewer items were recalled).

In the fixation condition the number of items recalled ranged from 4 to 11 with a mean of 9 , and in the scanning condition from 9 to 12 with a mean of 11 . The recall latency ranged from 3.3 to $15.0 \mathrm{sec}$ with a mean of $9.3 \mathrm{sec}$ in the fixation condition, and from 1.9 to $12.2 \mathrm{sec}$ with a mean of $4.5 \mathrm{sec}$ in the scanning condition. All Ss showed longer latencies when they were not allowed to scan during recall. The numbers of correct recalls were greater during scanning recall for seven Ss and equal for three Ss, but these ties were accompanied by increased differences in recall latencies. There were also instances of nearly equal recall latencies, but these were accompanied by larger differences in numbers of items recalled.

\section{Subject Reports}

At the conclusion of the experiment, Ss were asked to compare the difficulty of the fixation- and scanning-task conditions. All Ss rated the fixation condition more difficult than the scanning condition.

Another report obtained from the Ss concerned the nature of the recall strategies adopted. In general, the reported strategy was to attempt to visualize items in their locations. Some Ss also attempted to impose organization on the basis of visual characteristics of items, such as forming locational subgroups of angular and rounded geometric shapes. No strategies relying on verbal characteristics of item names were reported.

\section{DISCUSSION}

In the experiment the 12 stimulus elements were presented one at a time to hinder the imposition of nonvisual organizational schemes. Geometric shapes were used for the same reason, their abstract quality being presumably more difficult to organize into verbal mnemonic schemes than more concrete stimulus items would have been. Verbal factors were inevitably present in the experiment, however, since geometric shapes were identified by name in recall. In general, it is difficult to separate the verbal and visual cognitive modes in order to study only one; all that can be done is to design an experiment so that the mode under study is the one primarily activated. Subject reports indicated that the visual mode was predominant in the present experiment.

The evidence of a relationship between eye movements and imagery obtained in the present experiment does not support the findings of some prior studies (Bower, 1972; Hale \& Simpson, 1970). Both of these studies involved images which contained only two elements to be organized into a relationship, and so, in spatial terms at least, the organization in these images was simple. In this simple situation, eye fixation may not have been as relevant as in the present experiment, where 12 elements had to be related to each other with spatially organized imagery that was necessarily more complex.

The involvement of eye fixation in imagery may or may not be functional in nature. Kahneman (1973) has characterized the elicitation of eye fixation in spatially organized imagery as a nonfunctional occurrence, a general spatial orientation toward the object of thought. In perception there is evidence that under some conditions variations in patterns of eye fixation are functionally central to the perceptual process, determining what is perceived (Hindmarch, 1973). The present experiment, by demonstrating that inappropriate eye fixation produces a disruptive effect on imaging, indicates that eye fixation may play an analogous functional role in certain imagery contexts.

Another possible explanation for the present finding is that attention directed to the center of the visual field in imagery has a detrimental effect on imaging in peripheral visual areas. An analogous phenomenon also occurs in perception; when perception tasks at the center and the periphery of the visual field compete, performance of the peripheral task suffers (Webster \& Haslerud, 1964).

In the present experiment, when appropriate eye fixations were permitted, a spatially organized visual stimulus was recalled 
faster and more completely than when appropriate eye fixations were not permitted. This tends to indicate that the role of eye fixation in the recall of spatially organized imagery is analogous to the role of eye fixation in perception.

\section{REFERENCES}

Bower, G. Mental imagery and associative learning. In L. Gregg (Ed.), Cognition in learning and memory. New York: Wiley, 1972 .

Hale, S. M., \& Simpson, H. M. Effects of eye movements on the rate of discovery and the vividness of visual images. Perception \& Psychophy sics, 1970, 9, 242-245.

Haslerud, G. M. Transfer, memory and creativity: After-learning as perceptual process. Minneapolis: University of Minnesota Press, 1972.

Hębb, D. O. Concerning imagery. Psychological Review, 1968, $75,466-477$.

Hindmarch, I. Eye movements and the perception of phenomenal causality. Psychologica Belgica, 1973, 13, 17-23. Kahneman, D. Attention and effort. Englewood Cliffs, New Jersey : Prentice-Hall, 1973.

Mackw orth, N. H. The wide-angle reflection eye camera for visual choice and pupil size. Perception \& Psychophysics, 1968, 3, 32-34.

Marks, D. F. Individual differences in the vividness of visual imagery and their effect on function. In P. Sheehan (Ed.), Function and nature of imagery. New York and London: Academic Press, 1972.

Marks, D. F. Visual imagery differences and eye movements in the recall of pictures. Perception \& Psychophysics, 1973, 14, 407-412.

Webster, R. G., \& Haslerud, G. M. Influence on extreme peripheral vision of attention to a visual or auditory task. Journal of Experimental Psychology, 1964, 68, 269-272.

Winer, B. J. Statistical principles in experimental design. New York: McGraw-Hill, 1967.

\section{NOTE}

1. Hall, D. C. Eye movements in scanning iconic imagery. In preparation, 1974.

(Received for publication February 4, 1974.)

\title{
Sexual arousal and physical aggression: The inhibiting influence of "cheesecake" and nudes
}

\author{
ROBERT A. BARON* \\ Purdue University, West Lafayette, Indiana 47907
}

\begin{abstract}
Male Ss participated in an experiment designed to investigate the hypothesis that mild levels of sexual arousal would serve to inhibit subsequent physical aggression. Consistent with this suggestion, Ss exposed to two types of erotic stimuli ("cheesecake" or nudes) directed shocks of significantly shorter duration to a confederate of the $\mathbf{E}$ than Ss exposed only to neutral pictures of scenery, furniture, and abstract art. An interpretation of these findings based on the suggestion that mild sexual arousal is pleasurable, and serves to induce considerable positive affect among Ss, was proposed.
\end{abstract}

It has frequently been contended that sexual and aggressive motives are intimately linked. For example, Freud (1933) noted that desires to hurt or be hurt by one's lover form a normal part of heterosexual relations. Similarly, Berne (1964) has suggested that the arousal of aggressive motives often serves to enhance sexual pleasure for both men and women. Empirical evidence for the existence of a close bond between sexual and aggressive drives has been obtained by Barclay (1971), who observed that the arousal of one of these motives is generally associated with increments in the other. Apparently, then sexual and agressive drives are indeed closely linked.

But what of the effect of heightened sexual arousal on

*Requests for reprints should be sent to the author, Department of Psychological Sciences, Purdue University, West Lafayette, Indiana 47907. The author wishes to express his sincere appreciation to Paul Bell for his aid in performing the statistical analyses, and to Pam Geick, Ed Gurausaks, Julie Nilson, and Pete Shepard for their able assistance in collection of the data. overt aggressive behavior? Are sexually aroused individuals actually more likely to engage in subsequent attacks against others than individuals not so aroused? The view that heightened emotional arousal, whatever its source, often facilitates overt aggression suggests that this will in fact be the case (see, e.g., Bandura, 1973). However, the results of two recent experiments conducted by the author (Baron, 1974; Baron \& Bell, 1973) indicate that opposite effects may actually be observed. More specifically, it was found in both of these investigations that moderate levels of sexual arousal (induced through exposure to Playboy nudes), led to significant reductions in the level of aggression demonstrated by angry male Ss.

The present experiment was designed to extend this previous work by determining whether levels of sexual arousal even milder than those induced among Ss in the Baron (1974) and Baron and Bell (1973) studies, would also be successful in producing such effects. More specifically, it sought to determine whether exposure to 